\title{
Preferential Location for Arterial Dissection Presenting as Golf-Related Stroke
}

M.H. Choi, J.M. Hong, J.S. Lee, D.H. Shin, H.A. Choi, and K. Lee

\begin{abstract}
SUMMARY: Golf-related stroke has not been systematically reviewed. The purpose of our study was to describe in detail this particular stroke syndrome. Seven patients were analyzed at a university hospital and 7 patients were reviewed from MEDLINE literature. General demographics, symptom onset, neurologic signs, radiologic findings, and outcome were investigated. A total of 14 patients including 7 patients from the MEDLINE search were analyzed; all were men, with a mean age of $46.9 \pm 12.8$ years. Symptom onset was classified as during the golf swing ( $n=9)$, unknown $(n=3)$, and after playing golf $(n=2)$. Most patients $(n=12)$ showed involvement of the vertebral artery and 2 patients showed involvement of the internal carotid artery $(P=.008)$. Nine dissections were found on the right side, 3 on the left side, and 2 were bilateral $(P=.046)$. Twelve patients had extracranial involvement and 2 patients had intracranial involvement $(P=$ .008). Seven patients returned to normal, 5 returned to independence, 1 had unknown status, and 1 died. The anatomic preference of golf-related craniocervical arterial dissection is associated with the extracranial and vertebrobasilar system with a right-sided tendency as the result of stereotypical rotational movement during a golf swing.
\end{abstract}

ABBREVIATION: VA = vertebral artery

A s more people golf, golf-related injuries have become more prevalent, even in amateur golfers. ${ }^{1-3}$ Golf-related injuries are caused by either overuse or mechanical trauma, with most neurologic injuries being confined to the vertebral column. ${ }^{3} \mathrm{Sev}-$ eral case series of cervicocranial artery dissection associated with golf playing have been reported recently and referred to as "golfer's stroke." ${ }^{3-6}$ Previous case studies have reported cervical artery dissection associated with sudden hyperextension or rotation of the neck, including painting a ceiling, coughing, vomiting, sneezing, receiving anesthesia, and during cardiopulmonary resuscitation. ${ }^{7-9}$ Cervical artery dissection caused by physical activity represents a coincidental environmental trigger, and certain risk factors such as connective tissue disorders or hyperhomocysteinemia are associated with this type of injury. ${ }^{10}$ Because the golf swing is a habitual body motion that includes rapid head and body rotation, it may lead to repetitive mechanical com-

Received April 16, 2013; accepted after revision June 3.

From the Department of Neurology, Ajou University School of Medicine, Suwon, South Korea (M.H.C., J.M.H., J.S.L.); Department of Neurology, Gachon Gill Medical Center, Gacheon University School of Medicine, Incheon, South Korea (D.H.S.); and Department of Neurosurgery, The University of Texas Health Science Center, Houston, Texas (H.A.C., K.L.).

Please address correspondence to Ji Man Hong, MD, PhD, Department of Neurology, School of Medicine, Ajou University, 5 San, Woncheon-dong, Yongtong-gu Suwon-si, Kyunggi-do, 442-749, South Korea; e-mail: dacda@hanmail.net

三 Indicates article with supplemental on-line table.

http://dx.doi.org/10.3174/ajnr.A3768 pression of the extracranial cervical arteries during each swing. ${ }^{2}$ We investigated the clinical characteristics and radiologic findings in patients with golf-related stroke from cervicocranial arterial dissection.

\section{MATERIALS AND METHODS}

We retrospectively analyzed computerized data of a prospective registry that included 5469 patients with ischemic stroke from March 2001 to June 2011. Seven patients had a history of acute ischemic stroke caused by cervicocranial arterial dissection associated with golfing. Acute ischemic stroke was confirmed by diffusion-weighted MR imaging of the brain. All patients underwent a battery of diagnostic studies, including intracerebral and extracerebral vessel studies, routine blood tests, and a cardiologic work-up (electrocardiogram, transthoracic echocardiography, 24-hour Holter monitoring). Comorbidities and autoimmune markers were investigated to exclude underlying vasculopathy. Various auto-antibody screening tests such as rheumatoid factor and antinuclear, antiphospholipid, and antineutrophil cytoplasmic antibodies were analyzed. In addition, a MEDLINE search was performed from 1990 to December 2010 by use of the search terms "golfer's stroke," "golf-induced stroke," and "golf-induced dissection." This study was approved by the local institutional review board of the hospital.

We investigated general demographics, personal history of golf playing, handedness, situations at symptom onset, neuro- 


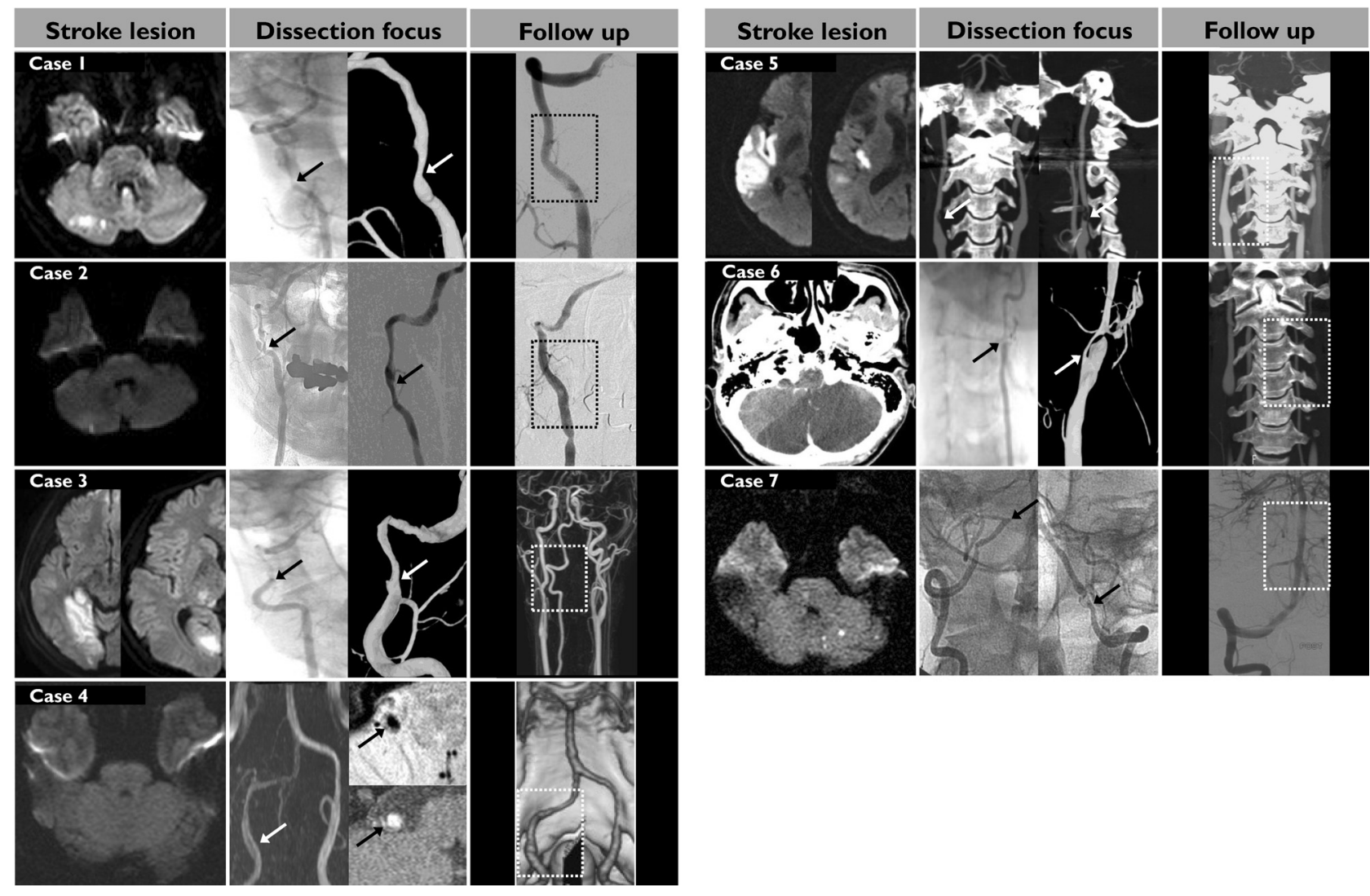

FIG 1. Radiologic findings (stroke location, dissection focus, and follow-up) of our 7 cases. Five cases had right-sided involvement: 3 patients (cases 1-3) with extracranial vertebral artery, 1 with intracranial VA (case 4), and 1 with extracranial carotid artery (case 5). One patient (case 6) had a left extracranial VA and 1 patient (case 7) had bilateral intracranial VA involvement. Black arrows indicate the dissection focus or foci. Dotted rectangles indicate follow-up images of dissection focus or foci.

logic signs and symptoms, treatment, and clinical outcomes. We also analyzed radiologic findings such as stroke location (anterior or posterior circulation), side of arterial dissection (right, left, or both), and anatomic location of the dissection (extracranial or intracranial). Status at symptom onset was classified as during swing, after golf exercise, and unknown. Clinical outcome was categorized as returned to normal ( $\mathrm{mRS}=0-1)$, independent $(\mathrm{mRS}=2-3)$, dependent $(\mathrm{mRS}=4-5)$, and death $(\mathrm{mRS}=6)$ at discharge. ${ }^{11}$ Differences between the stroke location, side of dissection, and anatomic location of the dissection were analyzed by use of the $\chi^{2}$ test. Statistical significance was considered at $P<.05$.

\section{RESULTS}

Fourteen male patients were examined. The demographic and clinical-radiologic findings are provided in the On-line Table. The mean age of the patients was $46.9 \pm 12.8$ years, which was younger than that of the general stroke population. Seven patients were right-handed. Of the 7 patients, 2 were professional golfers with 15-17 years of experience, and the remaining patients were amateur players with playing experience for approximately 7 years (range, $0.1-30$ years). None of the patients had a history of hypertension, diabetes, or autoimmune disorder. Symptom onset occurred during the golf swing $(n=9)$, at an unknown time $(n=$ $3)$, or after golf playing $(n=2)$. Twelve patients had posterior circulation symptoms such as vertigo, nystagmus, and body tilt- ing. Eight patients had localized pain at symptom onset. A potential source for vasculopathies and cardioembolic stroke was excluded from laboratory and cardiologic studies.

The location of arterial dissection was confirmed by cerebral angiography $(n=11)$, MR angiography $(n=2)$, and Doppler ultrasound $(n=1)$. The imaging studies revealed that 12 patients had involvement of the vertebral artery (VA) and 2 patients had ICA involvement $(P=.008)$. Nine patients had arterial dissections on the right side, of which 2 had ICA involvement; 3 had left side involvement; and 2 had bilateral lesions $(P=.046)$. There were 12 extracranial and 2 intracranial cases $(P=.008)$. Normal activity was possible in 7 patients, but the other cases revealed 5 independent patients and 1 death (On-line Table). Radiologic findings (stroke location and dissection focus) of our 7 cases are shown in Fig 1.

\section{DISCUSSION}

Our study illustrates that arterial dissection from golf-related stroke was more likely to be on the right side and predominantly in the extracranial vertebrobasilar system. This preference may be explained by the anatomic vulnerability of the vertebrobasilar system and the biomechanics of the golf swing.

Recent studies on ethnic differences in spontaneous vertebral artery dissection have shown that intracranial dissection is more common than extracranial dissection in East Asian populations. ${ }^{7}$ Our study of golf-related vertebral artery dissection shows that 


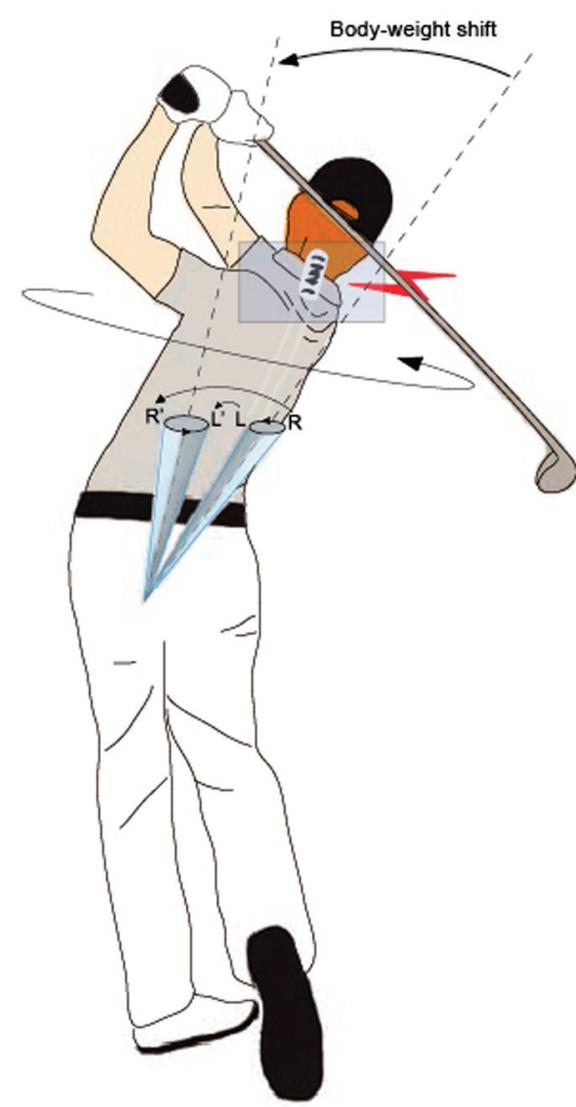

A

FIG 2. Mechanical explanation for the predominance of the right vertebral artery (VA) dissection during a golf swing. A, Late followthrough phase. $B$, Anatomy of neck muscles adjacent to the hypermobile segment (V3) of the VA. C, Vulnerability of the right VA during the golf swing. In a right-handed swing, there is a combination of right-to-left body-weight shift and counter-clockwise body rotation. This gives the right shoulder ( $R$ to $\left.R^{\prime}\right)$ a longer arch compared with the left shoulder ( $L$ to $\left.L^{\prime}\right)$. In the same setting of force ( $F$ ), the right VA receives a greater torque $(\tau)$ than does the left VA because it has a longer distance $(r)$ from the central axis (dotted line). The anatomic preference for this specific stroke syndrome can be explained by a momentary high torque $(\tau=r \times F)$ during body-weight shift and forceful rotation of the body and neck.

extracranial involvement is more frequent than intracranial involvement. As compared with either their intracranial segments or extracranial arteries of similar caliber, greater mobility of the extracranial segments of the carotid and vertebral arteries leads to increased susceptibility for injury by surrounding hard structures including bones, ligaments, and contracted muscles. ${ }^{6,8}$ Even with an external elastic lamina and a thickened media in the extracranial arteries, various studies have found that the atlantic (V3) portion of the vertebral artery is susceptible to VA dissection during trauma. ${ }^{8}$ This V3 portion is exposed without the support of bony structures and rotates freely during inadvertent head movement. Extracranial vertebral artery dissection has also been reported in patients who have undergone chiropractic manipulation, fixed neck posture caused by long-lasting surgery, or chest compressions during cardiopulmonary resuscitation. ${ }^{9,10}$ In addition, dynamic imaging studies show that the VA is compressed and even occluded at the extracranial portion during contralateral head rotation. ${ }^{12,13}$ These reasons support direct mechanical injury of the extracranial vessels as the etiology for golf-related stroke.

VA injuries during sports activities with repetitive neck movement have been reported in yoga, tennis, volleyball, judo, bowl- ing, and wrestling. ${ }^{3,14,15}$ Most sports-related vessel damage is caused by dissection or thromboembolism. ${ }^{3}$ A similar case of right VA dissection associated with unidirectional stereotypical movement was reported in a right-handed archer and is known as "bow hunter's stroke." ${ }^{15}$ As compared with other sports, the golf swing is a high-speed, unidirectional, stereotypical rotational movement. Force is proportional to mass and acceleration of an object, as stated by Newton's second law. Torque ( $\tau$ : torque) is a moment of force in rotating movement, and its magnitude depends on the length of the lever arm ( $r$ : length) and the force ( $F$ : force). Therefore, the driving torque of the golf swing is calculated as the cross-product of the distance and force, which tends to generate rotation, as expressed in the following equation: $\tau=r \times$ $F .{ }^{16}$ Our patients were all right-handed. In a right-handed swing, the central axis (hub) of the right leg during the back swing is changed to the left leg because of the body-weight shift from right to left to obtain forceful impact of the ball. As compared with the left VA, the right VA is more distant from the central axis during the body-weight shift and receives a greater torque during the golf swing. This swing trajectory also causes repetitive mechanical stress to the adjacent tissues including muscles, ligaments, and 
vessels. ${ }^{6}$ The increased stress of neck muscles adjacent to the right VA can lead to vessel injury by acting as a hammer when a golfer has greater torso-pelvic separation, misses the shot, or has insufficient warm-up. ${ }^{17}$ In the present study, symptoms occurred during the golf swing in many of the cases (On-line Table). ${ }^{18}$ Our 2 professionals and 1 player with low handicap self-reported attempts to maximize torso-pelvic separation to get more distance. From this perspective, a right-sided preference for this specific type of stroke can be explained by a momentary high torque status in relation to the forceful hitting phases (downswing, impact, and follow-through) and an anatomic vulnerability at the hypermobile portion of the right VA (Fig 2).

Our study has several limitations. Given the small number of patients with golf-related stroke in this study, investigations with more cases are needed for a balanced interpretation. Moreover, the handedness of half of the patients was unknown despite the significant association with the laterality of the golfer's stroke. Finally, video analyses of swing phases were not investigated in a biomechanical laboratory. Five cases (36\%) had no relationship with the direct golf swing even though the mechanical injury was suspicious for the development of arterial dissection during the swing.

\section{CONCLUSIONS}

Golf-related craniocervical arterial dissection occurs most often in the right extracranial vertebrobasilar system. Possible mechanisms are high mobility of the extracranial portion of the VA against a head rotation and mechanical injury to the respective vessels associated with high-speed, unidirectional, and stereotypical body rotation. Future studies are required not only to investigate detailed observations of the swing phase but to focus on other nonmechanical factors such as insufficient warm-up and incorrect posture.

\section{REFERENCES}

1. McCarroll JR. The frequency of golf injuries. Clin Sports Med 1996;15:1-7
2. Theriault G, Lachance P. Golf injuries: an overview. Sports Med 1998;26:43-57

3. Maroon JC, Gardner P, Abla AA, et al. "Golfer's stroke": golf-induced stroke from vertebral artery dissection. Surg Neurol 2007;67:163-68

4. Choi KD, Oh SJ, Yang TI, et al. Golfer's stroke from internal carotid artery dissection. Arch Neurol 2008;65:1122-23

5. Guptha SH, Promnitz AD, Warner A, et al. A 'collapsing' golfer. Cerebrovasc Dis 2005;19:281-82

6. Hong JM, Kim TJ, Lee JS. Neurological picture: repetitive internal carotid artery compression of the hyoid: a new mechanism of golfer's stroke? J Neurol Neurosurg Psychiatry 2011;82:233-34

7. Kim BM, Kim SH, Kim DI, et al. Outcomes and prognostic factors of intracranial unruptured vertebrobasilar artery dissection. Neurology 2011;76:1735-41

8. Schievink WI. Spontaneous dissection of the carotid and vertebral arteries. N Engl J Med 2001;344:898-906

9. Sherman DG, Hart RG, Easton JD. Abrupt change in head position and cerebral infarction. Stroke 1981;12:2-6

10. Tettenborn B, Caplan LR, Sloan MA, et al. Postoperative brainstem and cerebellar infarcts. Neurology 1993;43:471-77

11. Wilson JT, Hareendran A, Grant M, et al. Improving the assessment of outcomes in stroke: use of a structured interview to assign grades on the modified Rankin Scale. Stroke 2002;33:2243-46

12. Choi KD, Shin HY, Kim JS, et al. Rotational vertebral artery syndrome: oculographic analysis of nystagmus. Neurology 2005; 65:1287-90

13. Sakaguchi M, Kitagawa K, Hougaku H, et al. Mechanical compression of the extracranial vertebral artery during neck rotation. $\mathrm{Neu}$ rology 2003;61:845-47

14. DeBehnke DJ, Brady W. Vertebral artery dissection due to minor neck trauma. J Emerg Med 1994;12:27-31

15. Sorensen BF. Bow hunter's stroke. Neurosurgery 1978;2:259-61

16. Sharp RS. On the mechanics of the golf swing. Proc $R$ Soc $A$ 2009;465:551-70

17. Myers J, Lephart S, Tsai YS, et al. The role of upper torso and pelvis rotation in driving performance during the golf swing. J Sports Sci 2008;26:181-88

18. Ono Y, Arai K, Nishino A, et al. Bilateral dissections of the vertebral arteries followed by MRI. Prog Comput Imaging 2000;22: 199-204 